\title{
Relaxation dynamics in the excited states of a ketocyanine dye probed by femtosecond transient absorption spectroscopy
}

\author{
JAHUR A MONDAL, SANDEEP VERMA, HIRENDRA N GHOSH and DIPAK K PALIT* \\ Radiation and Photochemistry Division, Bhabha Atomic Research Centre, Mumbai 400085 \\ e-mail: dkpalit@barc.gov.in
}

\begin{abstract}
Relaxation dynamics of the excited singlet states of 2,5-bis-(N-methyl-N-1,3-propdienylaniline)-cyclopentanone (MPAC), a ketocyanine dye, have been investigated using steady-state absorption and emission as well as femtosecond time-resolved absorption spectroscopic techniques. Following photoexcitation using $400 \mathrm{~nm}$ light, the molecule is excited to the $S_{2}$ state, which is fluorescent in rigid matrices at $77 \mathrm{~K} . \mathrm{S}_{2}$ state is nearly non-fluorescent in solution and has a very short lifetime $(0 \cdot 5 \pm 0 \cdot 2 \mathrm{ps})$. In polar aprotic solvents, the $\mathrm{S}_{1}$ state follows a complex multi-exponential relaxation dynamics consisting of torsional motion of the donor groups, solvent re-organization as well as photoisomerization processes. However, in alcoholic solvents, solvent re-organization via intermolecular hydrogen-bonding interaction is the only relaxation process observed in the $\mathrm{S}_{1}$ state. In trifluoroethanol, a strong hydrogen bonding solvent, conversion of the non-hydrogen-bonded form, which is formed following photoexcitation, to the hydrogen-bonded complex has been clearly evident in the relaxation process of the $\mathrm{S}_{1}$ state.
\end{abstract}

Keywords. Ketocyanine dye; excited state dynamics; $\mathrm{S}_{2}$-fluoroscence; photoisomerization; solvent reorganization.

\section{Introduction}

Photophysical and spectroscopic properties of the ketocyanine dyes have been the subject of intensive investigations in recent years. ${ }^{1-6}$ The pronounced solvent effects in both absorption and emission spectra of these dyes make them promising probes for monitoring micro-polarity, hydrogen-bond donating interaction, metal ion sensing, investigation of the cell membrane structures, evaluating the micro-environmental characteristics of biochemical and biological systems and many others. ${ }^{1,2,5-10}$ For example, Lobnik and co-workers have used MPAC to probe the micropolarity of various solvents and ormosils (organically modified siloxanes). ${ }^{7 \mathrm{c}}$ They observed remarkable changes in the fluorescence band positions and/ or intensities as a function of the polarity of the ormosil. Some of them are also used as laser dyes or photosensitizers and have found several industrial applications in photopolymer imaging systems. ${ }^{11-13}$ Crown ether substituted derivative of ketocyanine dyes are efficient fluorophores for alkaline earth cation recognition. ${ }^{14}$

In addition, electron donor-acceptor (EDA) substituted $\pi$-conjugated polyenes have been of primary importance in the search of optical materials. Exten-

\footnotetext{
*For correspondence
}

sive research has been carried out on polymethine cyanine dyes for high density optical recording, ${ }^{15}$ carotenoids for mimicking natural photosynthesis, ${ }^{16}$ and push-pull polyenes with EDA end groups for non-linear optics, harmonic generation, optical limitation as well as optical imaging, because of their high polarizability. ${ }^{17}$ However, although ketocyanine dyes have been found their applications in many other areas, feasibility of their applications as optical materials is yet to be explored. For this purpose, information regarding the dynamics of the excited states of these dyes is of primary importance.

Recently, we reported our results on the dynamics of the excited singlet (both the $S_{2}$ and $S_{1}$ ) states of a ketocyanine dye, namely, 2,5-bis-[(2,3-dihydroindolyl)propylene]-cyclopentanone (KCD), in different kinds of media using femtosecond transient absorption spectroscopic technique. ${ }^{18}$ Following photoexcitation of KCD to its $\mathrm{S}_{2}$ state using $400 \mathrm{~nm}$ light, we observed $\mathrm{S}_{2}$ fluoresence in the case of ketocyanine dyes. Time-resolved measurements of the dynamics of the excited states in both aprotic and protic solvents revealed the presence of an ultrafast component in the decay dynamics of the $S_{1}$ state. A good correlation between the lifetime of this component and the longitudinal relaxation times $\left(\tau_{\mathrm{L}}\right)$ of the solvents suggested that this component arose due to solvation in polar solvents. More significant evolution of the 
spectroscopic properties of the $\mathrm{S}_{1}$ state in alcoholic solvents in the ultrafast time domain has been explained by the occurrence of the re-positioning of the hydrogen bonds around the carbonyl group in the excited state of KCD. In this study, we present our results on the photophysical properties and relaxation dynamics of the excited states of another ketocyanine dye, 2,5bis-(N-Methyl-N-1,3-Propdienylaniline)-cyclopentanone (MPAC). Its structure is shown in scheme 1.

Along with other ketocyanine dyes, Bagchi and his co-workers also studied the photophysical and solvatochromic behaviour of MPAC using steadystate absorption, fluorescence and time-resolved fluorescence measurements. ${ }^{2}$ They also showed that while non-specific dipolar solvation is responsible for the solvatochromic properties of these dyes in aprotic solvents, specific interaction involving formation of intermolecular hydrogen bond provides stronger solvatochromic behaviour of these dyes in protic solvents. ${ }^{2 \mathrm{i} 7 \mathrm{a}}$ This group as well as Pivovarenko et al have shown that the ketocyanine dyes form strong hydrogen-bonded complexes both in the ground state as well as in the excited state. ${ }^{2 a, 6}$ To explain their observation of a blue shift of the absorption maximum of a similar ketocyanine dye in rigid matrices at $77 \mathrm{~K}$ as compared to that at $298 \mathrm{~K}$, Pramanik et al proposed the twisted intramolecular charge transfer (TICT) process in the $\mathrm{S}_{1}$ state, which is inhibited or slowed down significantly in rigid matrices. ${ }^{2 \mathrm{~h}}$ On the other hand, Doroshenko and Pivovarenko proposed that the change of configuration due to cis-trans photoisomerization about one of the flexible $\mathrm{C}=\mathrm{C}$ bonds may also provide another possible channel for the relaxation process of the dye in the excited state. ${ }^{4}$ To unravel the possible role of these photophysical processes in the excited state relaxation dynamics of this class of dyes, we became motivated to investigate the excited state dynamics of MPAC using ultrafast transient absorption spectroscopic technique.

\section{Experimental}

MPAC was a gift from Prof Sanjib Bagchi of Burdwan University, India. The method of synthesis and puri-

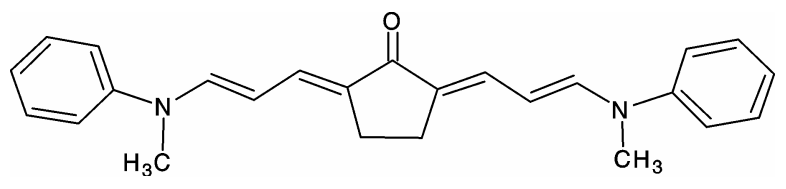

Scheme 1. Chemical Structure of MPAC. fication of this dye has been described elsewhere. ${ }^{2 a}$ All the solvents used were of spectroscopic grade (Spectrochem, India) and used as received without further purification.

Steady-state absorption spectra were recorded using a Shimadzu model UV-160A spectrophotometer. Fluorescence spectra were recorded using Hitachi model 4010 spectrofluorimeter. The emission spectra were recorded using $400 \mathrm{~nm}$ light for photoexcitation and the samples were kept in a quartz tube of $4 \mathrm{~mm}$ diameter, which was put inside a quartz debar flask. For measuring the fluorescence spectra in solid matrices at liquid nitrogen temperature $(77 \mathrm{~K})$, the samples in the quartz tube were immersed into liquid nitrogen taken in the quartz debar flask. Both the emission spectra at room temperature and $77 \mathrm{~K}$ were recorded in the same experimental configuration.

Relaxation processes in the sub- $1 \mathrm{~ns}$ time-domain have been measured using a femtosecond pumpprobe transient absorption spectrometer, which used a femtosecond Ti : Sapphire laser system supplied by CDP, Russia. The laser system consists of a Ti: Sapphire laser oscillator (model TISSA-50), which is pumped by a 5 Watt diode-pumped solid state laser and produces laser pulses of $6 \mathrm{~nJ}$ energy at $800 \mathrm{~nm}$. These pulses are amplified in an optical amplifier (model MPA-50) to generate $70 \mathrm{fs}$ laser pulses of about $300 \mu \mathrm{J}$ energy at a repetition rate of $1 \mathrm{kHz}$ using the chirped pulse amplification (CPA) technique. Pump pulses at $400 \mathrm{~nm}$ with an energy of $5 \mu \mathrm{J} /$ pulse are generated for excitation of the samples by frequency-doubling of one part of the $800 \mathrm{~nm}$ output of the amplifier in a $0.5 \mathrm{~mm}$ thick BBO crystal and the other part of the amplifier output is used to generate the white light continuum $(470-1000 \mathrm{~nm})$ probe in a $2 \mathrm{~mm}$ thick sapphire plate. The direction of polarization of the pump beam is fixed at the magic angle with respect to the probe beam. The sample solutions are kept flowing through a quartz cell of $1 \mathrm{~mm}$ path length. The probe beam is split into two equal parts, one of which passed through the excited zone of the sample to fall onto an integrating photo-diode and the other part reaches directly to another photodiode. For monitoring the decay dynamics at a particular wavelength region with $10 \mathrm{~nm}$ bandwidth is selected using a pair of interference filters placed in front of the photodiodes. The variation of the relative intensities of the two probe beams at different delay-times with respect to the pump beam are monitored using the photodiodes coupled with two different boxcar integrators and then to an ADC and 
computer. The overall time resolution of the absorption spectrometer has been determined to be about $120 \mathrm{fs}$ by measuring the ultrafast growth of excited state absorption (ESA) for perylene in cyclohexane at $690 \mathrm{~nm}$. The temporal profiles recorded using different probe wavelengths are fitted with up to three exponentially decaying or growing components by iterative deconvolution method using a $\operatorname{sech}^{2}$ type instrument response function with fwhm of $120 \mathrm{fs}$.

\section{Results}

\subsection{Absorption and emission studies}

Bagchi and his co-workers have already reported the steady-state absorption and fluorescence characteristics of MPAC in different kinds of solvents. ${ }^{2}$ However, since we have used $400 \mathrm{~nm}$ laser light as an excitation source to study the ultrafast relaxation dynamics of the excited states of this molecule, it is important to understand the steady state absorption and fluorescence spectroscopic properties of it following photoexcitation using $400 \mathrm{~nm}$ light. Figure 1 shows

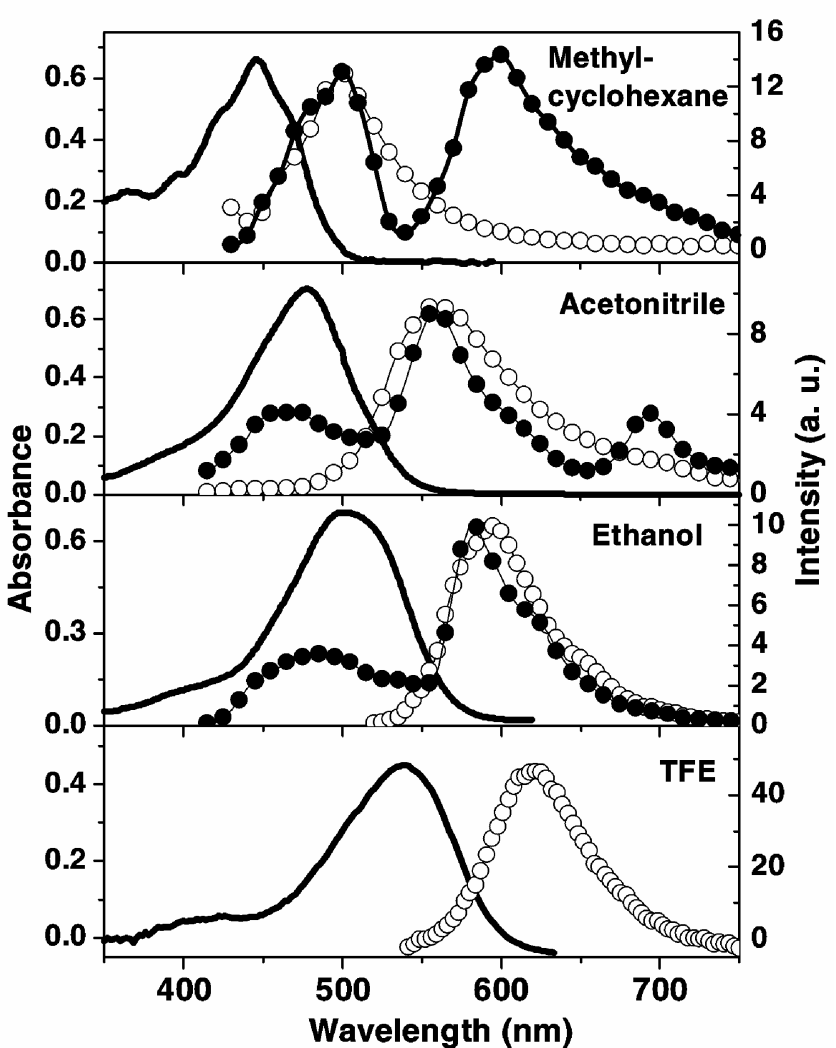

Figure 1. Ground-state absorption spectra (solid black line) and fluorescence spectra of MPAC recorded in different solvents using $400 \mathrm{~nm}$ photoexcitation at room temperature (open circles) and in solid matrices at $77 \mathrm{~K}$ (solid circles). these spectra recorded in four different kinds of solvents, namely, methylcyclohexane (non-polar aprotic), acetonitrile (polar aprotic), ethanol (polar protic) and trifluoroethanol (TFE, which is a strong hydrogen-bonding solvent). In each of these solvents, the absorption spectrum recorded in the $350-600 \mathrm{~nm}$ region consists of a strong band in the 450-600 nm region because of the $S_{1} \leftarrow S_{0}$ transition, and another absorption band, which appears as a shoulder of this band in the $350-450 \mathrm{~nm}$ region could be assigned to the $\mathrm{S}_{2} \leftarrow \mathrm{S}_{0}$ transition (vide infra).

In methylcyclohexane, the absorption maximum of the lowest energy absorption band appears at $447 \mathrm{~nm}\left(22371 \mathrm{~cm}^{-1}\right)$ and those in acetonitrile, ethanol and TFE are at $478 \mathrm{~nm}\left(20920 \mathrm{~cm}^{-1}\right), 505 \mathrm{~nm}$ $\left(19801 \mathrm{~cm}^{-1}\right)$ and $538 \mathrm{~nm}\left(18587 \mathrm{~cm}^{-1}\right)$, respectively. This large bathochromic shift of this absorption band with increasing solvent polarity indicates the intramolecular charge transfer (ICT) nature of the lowest energy absorption band. Larger bathochromic shift of the absorption band in ethanol and TFE as compared to that in acetonitrile in spite of larger dielectric polarity of acetonitrile as compared to those of ethanol and TFE suggests the formation of hydrogen-bonded complex between the protic solvent and MPAC in the ground state. Additionally, the flattop absorption band in ethanol suggests the existence of an equilibrium between the non-hydrogenbonded and hydrogen-bonded molecules in solution. In TFE, however, the shape of the absorption spectrum suggests that the equilibrium is in favour of the hydrogen-bonded complex.

The emission spectra of MPAC have been recorded in all these solvents both in solution at room temperature $(298 \mathrm{~K})$ and in rigid matrices at $77 \mathrm{~K}$ following photoexcitation using $400 \mathrm{~nm}$ light and they are also shown in figure 1. The emission spectrum recorded in each of these solvents at room temperature, consists of a single band and the maximum of this band shifts from $500 \mathrm{~nm}\left(20,000 \mathrm{~cm}^{-1}\right)$ in methylcyclohexane to $560 \mathrm{~nm}\left(17857 \mathrm{~cm}^{-1}\right)$ in acetonitrile, $595 \mathrm{~nm}\left(16,807 \mathrm{~cm}^{-1}\right)$ in ethanol and $620 \mathrm{~nm}$ $\left(16129 \mathrm{~cm}^{-1}\right)$ in TFE. This suggests more polar nature of the emitting excited state than the ground state. Additionally, emission spectrum is more redshifted in ethanol and TFE as compared to that in acetonitrile, suggesting the role of hydrogen-bonding interaction in solvation of the excited state. It is important to note that the $\mathrm{S}_{1}$-fluorescence band in acetonitrile solution at room temperature is broad with a long tail in the lower energy region. FWHM of this band $\left(2787 \mathrm{~cm}^{-1}\right)$ is larger than those of the $S_{1}$ - 
fluorescence band recorded in methylcyclohexane $\left(2479 \mathrm{~cm}^{-1}\right)$ and ethanol $\left(1889 \mathrm{~cm}^{-1}\right)$ at room temperature. However, FWHM of the $S_{1}$-fluorescence band in acetonitrile matrix at $77 \mathrm{~K}\left(1730 \mathrm{~cm}^{-1}\right)$ is significantly reduced as well as the long tail in the lower energy region of the spectrum is also absent in this spectrum. These facts suggest that the MPAC molecules undergo some kind of conformational relaxation in acetonitrile solution, which is not observed in the rigid matrix. Emission from different conformers is not very evident in methylcyclohexane and ethanol solutions. It is important to note that although the fluorescence maximum undergoes a large red shift in acetonitrile, ethanol and TFE as compared to that in methylcyclohexane, Stokes shift values, which are $2371,3063,2994$ and $2458 \mathrm{~cm}^{-1}$ in these four solvents, respectively, do not increase significantly in more polar solvents. The change of dipole moment $(\Delta \mu)$ between the ground state $\left(\mathrm{S}_{0}\right)$ and the emitting $\mathrm{S}_{1}$ state has been determined in aprotic solvents using modified Lippert-Mataga equation and this value is $1.5 \mathrm{D}^{2 \mathrm{e}}$ Hence, the dynamic shift of the stimulated emission maximum in the time-resolved spectra because of polar salvation is expected to be small (vide infra).

The emission spectrum recorded in rigid matrices of methylcyclohexane at $77 \mathrm{~K}$ shows the appearance of an additional emission band in the $550-750 \mathrm{~nm}$ region with maximum at $600 \mathrm{~nm}$, which can be assigned to phosphorescence emission from the triplet $\left(T_{1}\right)$ state ${ }^{18}$ In rigid matrices of acetonitrile at $77 \mathrm{~K}$, however, in addition to the normal $\mathrm{S}_{1}$ fluorescence band in the 515-615 $\mathrm{nm}$ region and the phosphorescence emission band in the $650-750 \mathrm{~nm}$ region, we observe the appearance of another emission band in the $420-500 \mathrm{~nm}$ region with maximum at $465 \mathrm{~nm}$. Since, the absorption spectrum of MPAC in acetonitrile clearly suggests that a photon of $400 \mathrm{~nm}$ light excites the molecule to its $S_{2}$ state, the higher energy emission band in the $420-500 \mathrm{~nm}$ region can be assigned to the $\mathrm{S}_{2}$-fluorescence. In ethanol matrix at $77 \mathrm{~K}$, only the $\mathrm{S}_{2}$-fluorescence and the $\mathrm{S}_{1}$-fluorescence bands are observed. However, in this medium, the maximum of the $S_{1}$-fluororescence band is shifted by about $15 \mathrm{~nm}$ as compared to that of the spectrum recorded in solution at room temperature. On the contrary, in aprotic solvents, the maximum of the $\mathrm{S}_{1}$-fluorescence band remains at the same place both in solution and rigid matrix, although the width of the fluorescence spectrum recorded in solution is broader than that recorded in rigid matrix. In rigid matrix, the solvent motions are frozen and the re-organization of the solvent molecules around the excited MPAC molecule is suppressed. No shift between the maxima of the $\mathrm{S}_{1}$-emission spectra recorded in solution and rigid matrix in acetonitrile suggests that the free energy of stabilization of the $\mathrm{S}_{1}$ state because of solvation is nearly negligible. However, about $15 \mathrm{~nm}$ shift in ethanol obviously suggests the hydrogen bonding interaction between the $\mathrm{S}_{1}$ state of MPAC and the protic solvent. ${ }^{2 \mathrm{i}, 7 \mathrm{a}, \mathrm{b}}$

Absence of phosphorescence emission in protic solvents suggests significantly lower yield of the intersystem crossing process. This has been explained by the fact that in protic solvents, the ${ }^{3} n v^{*}$ and ${ }^{3} \pi \pi^{*}$ states reverse their positions in the energy scale so that the $\mathrm{T}_{1}$ and $\mathrm{T}_{2}$ states achieve the $\pi \pi^{*}$ and $n \pi^{*}$ characters, respectively. According to El-Sayed's rule, the intersystem crossing (ISC) from $\left.\mathrm{S}_{1} \pi \pi^{*}\right) \rightarrow$ $\mathrm{T}_{1}\left(\pi \pi^{*}\right)$ is not so efficient and $\left.\mathrm{S}_{1} \pi \pi^{*}\right) \rightarrow \mathrm{T}_{2}\left(n \pi^{*}\right)$ becomes a thermally activated process and hence the rate of ISC decreases in protic solvent as compared to that in an aprotic solvent of similar polarity. 19,20 Conformational relaxation in aprotic solvents may possibly be another reason for efficient radiationless deactivation of the $S_{1}$ state.

\subsection{Femtosecond transient absorption studies}

Figure 2 presents the time-resolved transient absorption spectra recorded following photoexcitation of MPAC in acetonitrile using $400 \mathrm{~nm}$ light pulses of

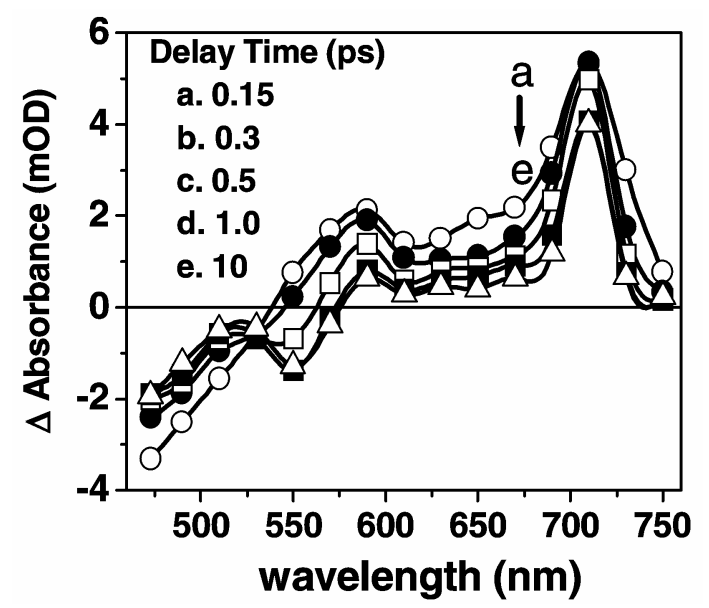

Figure 2. Time-resolved transient absorption spectra constructed at different delay-times in sub-10 ps timedomain following photoexcitation of MPAC in acetonitrile using $400 \mathrm{~nm}$ laser pulses of $50 \mathrm{fs}$ duration. 

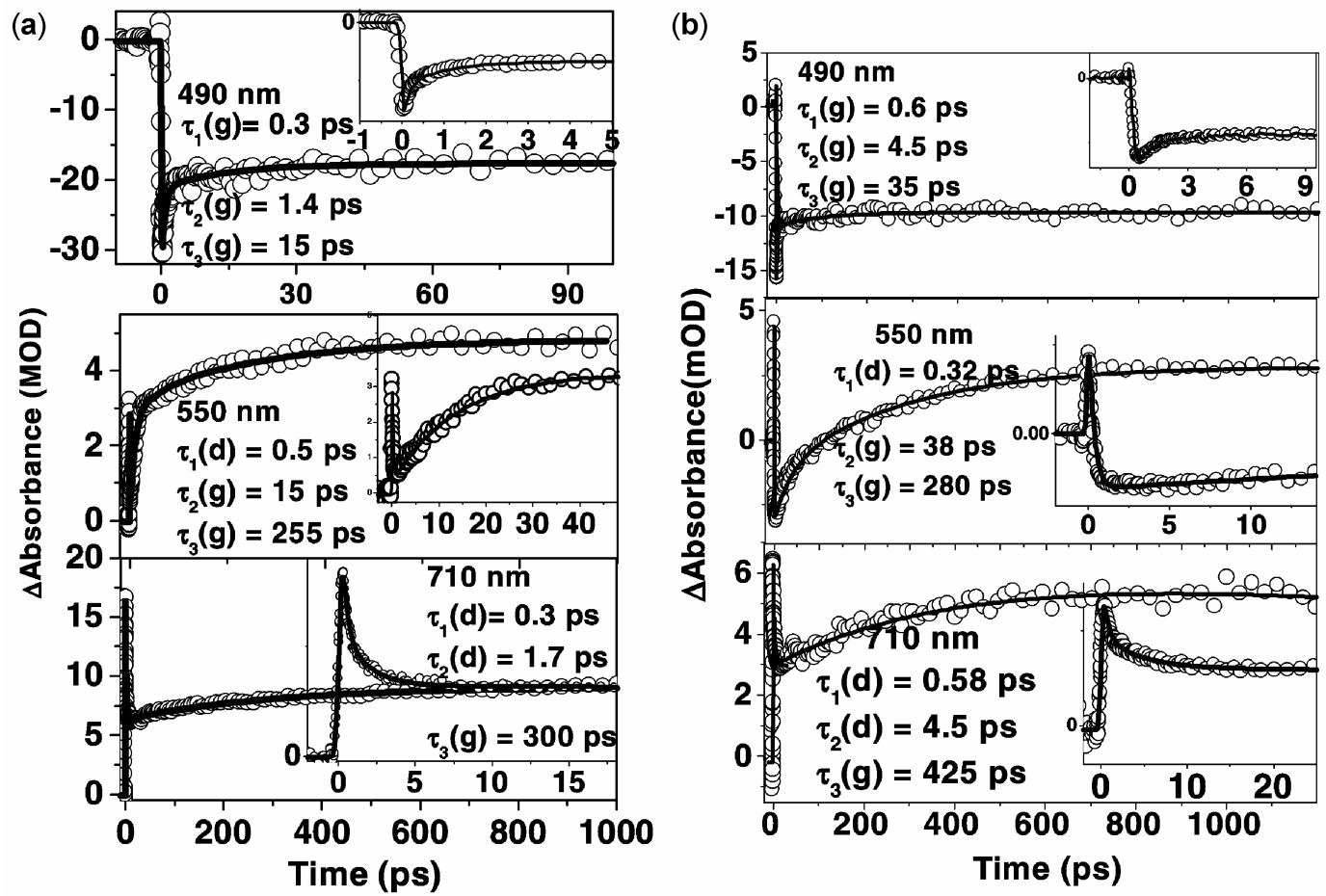

Figure 3. Temporal profiles of the transients monitored at three different wavelengths following photoexcitation of MPAC in acetonitrile (a) and DMSO (b) along with the best-fit function (solid lines). Lifetimes of the different components are also shown in the figure.

$50 \mathrm{fs}$ duration. The spectrum recorded at $0 \cdot 15 \mathrm{ps}$ delay-time shows two excited state absorption (ESA) bands in the $550-750 \mathrm{~nm}$ region with maxima at 590 and $710 \mathrm{~nm}$ and a negative absorption band in the $470-550 \mathrm{~nm}$ region. As mentioned in the previous section ( $\$ 3.1)$ that the MPAC molecule is excited to the $\mathrm{S}_{2}$ electronic state following absorption of a photon of $400 \mathrm{~nm}$ wavelength, the two ESA bands can be assigned to transitions from the $S_{2}$ state to the higher electronic states and the negative absorption band in the 470-530 nm region may have arisen due to ground state bleaching (since the ground state of MPAC has absorption below $530 \mathrm{~nm}$ ), or it may have the contributions from both the ground state bleaching as well as stimulated emission (SE) from the $\mathrm{S}_{2}$ state (see figure 1).

With increasing the delay time up to $2 \mathrm{ps,} \mathrm{the}$ negative absorption band in the blue region (below $510 \mathrm{~nm}$ ) recovers by about $30 \%$ and the ESA band in the $670-750 \mathrm{~nm}$ region also decays nearly to the same extent. However, the ESA band in the 590$650 \mathrm{~nm}$ region decays significantly. Decay of ESA in the 540-570 $\mathrm{nm}$ region has even resulted in the appearance of a new negative absorption band in this region with a maximum at ca $550 \mathrm{~nm}$ and an isos- bestic point at $525 \mathrm{~nm}$. Considering the fact that the $\mathrm{S}_{1}$-fluorescence spectrum has the maximum at $560 \mathrm{~nm}$, this negative absorption band with maximum at ca $550 \mathrm{~nm}$ can possibly be assigned to $\mathrm{SE}$ from the $\mathrm{S}_{1}$ state. ${ }^{18,19,21}$ The isosbestic point, which occurred following the partial recovery of the negative absorption band in the 470-510 nm region and the growth of SE in the 530-570 nm region, suggests that the major fraction of the population in the $\mathrm{S}_{2}$ state, created due to photoexcitation, decays mainly to populate the $S_{1}$ state through the internal conversion (IC) process, because of low quantum efficiency of the $\mathrm{S}_{2}$-fluorescence in solution at room temperature as compared to that of the IC process from the $S_{2}$ to the $\mathrm{S}_{1}$ state. Therefore, the decay of the negative absorption band in the $470-530 \mathrm{~nm}$ region may be assigned to the decay of the intensity of SE from the $S_{2}$ state rather than the bleaching recovery due to $S_{2} \rightarrow S_{0}$ fluorescence.

We recorded the time-resolved spectra up to $10 \mathrm{ps}$ delay-time. However, the temporal profiles recorded up to $1.2 \mathrm{~ns}$ delay-time at a few selective wavelengths, e.g. at 490, 550 and $710 \mathrm{~nm}$ (figure 3), reveal that the ultrafast evolution of the characteristics of the transient species, as revealed by the time- 
resolved transient spectra presented in figure 2 , is followed by a much slower process taking place in sub-nanosecond time-domain. Temporal profiles at these three wavelengths have also been recorded in DMSO and have been seen to follow similar dynamical characteristics as observed in acetonitrile (figure $3 b)$.

Multi-exponential analysis of these temporal profiles provide information regarding the lifetime of the $S_{2}$ state, to which the molecules are excited by $400 \mathrm{~nm}$ photon as well as the dynamics of the relaxation processes taking place in the $\mathrm{S}_{1}$ state. At $490 \mathrm{~nm}$, intensity of SE from the $S_{2}$ state increases within the instrument response time and then decays tri-exponentially. While the lifetime of the third component is reasonably long (longer than a few nanosecond), the lifetimes of the shorter-lived components are 0.8 and $15 \mathrm{ps}$, in acetonitrile and 1.2 and $35 \mathrm{ps}$ in DMSO (table 1). At $550 \mathrm{~nm}$, following the rise of ESA with the instrument response time, it decays very fast to reach a negative absorption value due to the development of an SE band in this region. At longer delay-time, SE also decays leading to biexponential growth of ESA, which has a lifetime of a few nanosecond. The lifetimes associated with these processes are $0 \cdot 5,15$ and 255 ps in acetonitrile and $0 \cdot 32,38$ and $280 \mathrm{ps}$ in DMSO, respectively. The temporal profiles recorded at $710 \mathrm{~nm}$ reveal the growth of ESA with the instrument response time, followed by a biexponential decay. However, ESA, in stead of decaying to zero value, shows another exponential growth to reach a steady value, which decays at a longer time-domain. The lifetimes of the two initial decay components and the subsequent growth component of ESA are $0.3,1.7$ and $300 \mathrm{ps}$ in acetonitrile and $0.6,4.5$ and 425 ps in DMSO, respectively.

These facts suggest that the relaxation dynamics of the $S_{1}$ state of MPAC in aprotic solvents are complex because of involvement of more than one transient species, which is the reason for wavelengthdependent temporal dynamics. Since the temporal profile recorded at $710 \mathrm{~nm}$ is free from overlapping effect of the bleaching and SE bands, this data will be useful to make an assignment of the decay and the growth components to different photophysical processes undergone by the excited states of MPAC in aprotic solvents. The ultrafast decay components with the lifetimes of $0.3 \mathrm{ps}$ in acetonitrile and $0.6 \mathrm{ps}$ in DMSO can be assigned to that of the $\mathrm{S}_{2}$ state because of the obvious reason that the molecules are excited to this state using $400 \mathrm{~nm}$ light. This component is also observed in the temporal profiles at other wavelengths too. Other decay or growth components observed at all the monitoring wavelengths are assigned to the relaxation processes undergone by the $\mathrm{S}_{1}$ state.

Figure 4 presents the time-resolved transient spectra recorded in ethanol. The spectrum recorded at $0 \cdot 15 \mathrm{ps}$ has two ESA bands in the $550-750 \mathrm{~nm}$ region and two negative absorption bands in the 470$530 \mathrm{~nm}$ region. With increasing the delay-time, intensities of all these bands decrease. Continuous decay of the SE band in the 510-530 nm region up to $100 \mathrm{ps}$ delay-time leads to the development of an ESA band with maximum at $530 \mathrm{~nm}$, while the decay of ESA band in the $550-700 \mathrm{~nm}$ region leads to the development of a broad SE band with maximum at ca $610 \mathrm{~nm}$.

Temporal profiles recorded at a few selective wavelengths are shown in figure 5. At $490 \mathrm{~nm}$, the negative absorption, following its growth with the instrument response time, decays triexponentially. Two of the three decay components are ultrafast having lifetimes of 0.8 and 20 ps and the third component is much longer lived, having lifetime in the order of a few nanosecond, which represent the decay of the $S_{1}$ state. ${ }^{2 e}$ Each of the temporal profiles recorded at other wavelengths in the $510-710 \mathrm{~nm}$ region could also be fitted with three components (decay and/or growth) with the lifetime values of $0.8 \pm 0.03 \mathrm{ps}, 20 \pm 2 \mathrm{ps}$ and a long one. However,

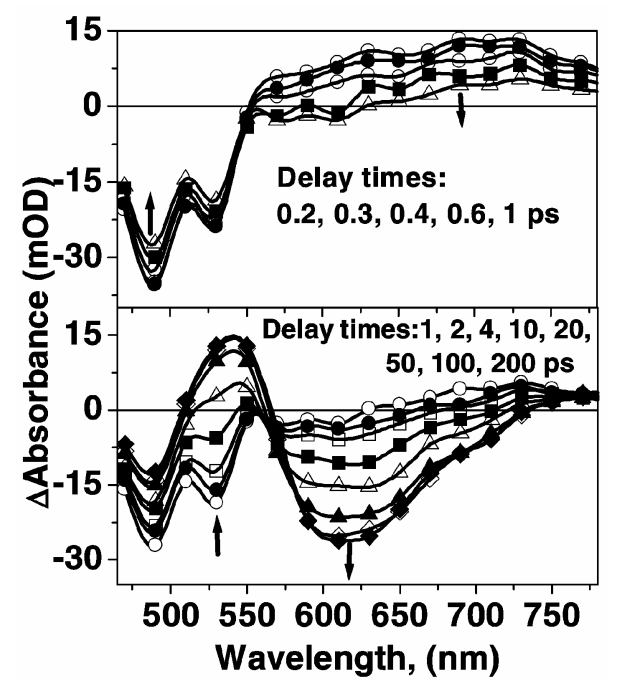

Figure 4. Time-resolved transient absorption spectra recorded following photoexcitation of MPAC in ethanol using $400 \mathrm{~nm}$ laser pulses of $50 \mathrm{fs}$ duration. 
although the decay profile of ESA recorded at the wavelengths longer than $750 \mathrm{~nm}$ also could be fitted with three decay components, the lifetime of the second component (12 ps) is much shorter as compared to that measured at shorter wavelengths.

We have recorded the temporal dynamics of the transient species created following photoexcitation of MPAC in a few other alcoholic solvents, namely, methanol, 1-propanol, 1-pentanol, 1-octanol and cyclohexanol at 490,610 and $710 \mathrm{~nm}$. The dynamical characteristics of the transient species in these solvents have been observed to be similar to those observed in ethanol and each of the temporal profiles recorded at these three wavelengths could be fitted with three exponential terms having the same lifetime values in a particular solvent. Lifetime of the first ultrafast component, which is assigned to that of the $S_{2}$ state, is more or less similar $\left(\tau_{1}=\right.$ $0.5 \pm 0 \cdot 1 \mathrm{ps}$ ) in each of these protic solvents. However, the lifetime of the second component $\left(\tau_{2}\right)$ increases monotonically as the solvent is changed from methanol to 1-octanol. In table 1 , values of

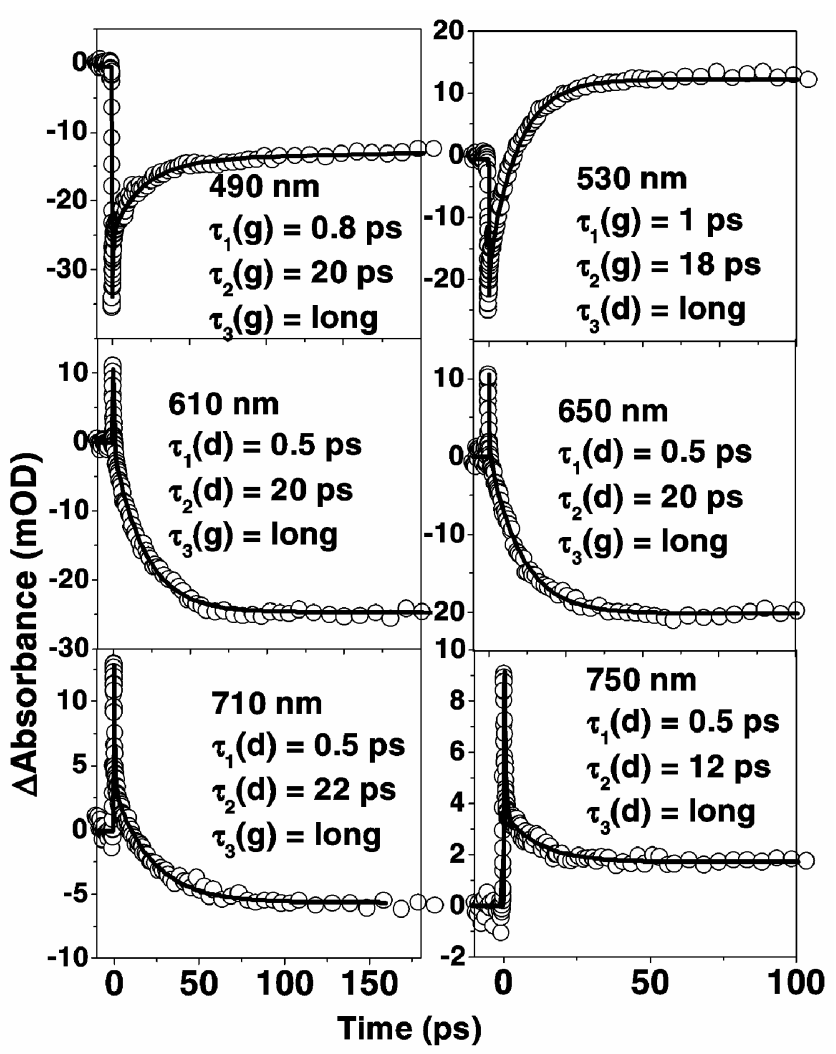

Figure 5. Temporal profiles of the transients monitored at a few selective wavelengths, following photoexcitation of MPAC in ethanol using $400 \mathrm{~nm}$ light, along with the best-fit functions (solid lines). Lifetimes of different components are also shown in figure. these lifetimes have been compared with the properties of the solvents, namely, viscosity, longitudinal relaxation time and average salvation time.

Figure 6 shows the time-resolved spectra of the transient species produced due to photoexcitation of MPAC in TFE with $400 \mathrm{~nm}$ laser pulses and the temporal dynamics monitored at different wavelengths are presented in figure 7 . The spectrum constructed for 0.15 ps delay-time consists of two negative absorption bands in $490-530 \mathrm{~nm}$ and $530-620 \mathrm{~nm}$ regions, and an ESA band in $620-800 \mathrm{~nm}$ region. All these features are the characteristics of the $S_{2}$ state of MPAC. Between the two negative absorption bands, the first one is assigned to the stimulated emission from the $S_{2}$ state and the other one is to the bleaching of the ground state on photoexcitation. The time-resolved spectra constructed at longer delay-times (up to $1 \mathrm{ps}$ ) reveal the rapid decay of the SE band in the 490-530 nm region because of the $\mathrm{S}_{2} \rightarrow \mathrm{S}_{0}$ radiative transition and the ESA band in the $620-800 \mathrm{~nm}$ region because of $S_{2} \rightarrow S_{1}$ internal conversion process. Increase of negative absorbance in the $590-730 \mathrm{~nm}$ region is also associated with the formation of the $S_{1}$ state because of the later process. Upon further increase in delay-time, the SE band with maximum at $575 \mathrm{~nm}$ decays with the concomitant growth of a new SE band with a maximum at $625 \mathrm{~nm}$. Since the fluorescence lifetime of the $S_{1}$ state is a few nanosecond, ${ }^{2 \mathrm{e}}$ this kind of evolution of the SE band suggests the conversion of one from of the $\mathrm{S}_{1}$ state to another form in the same state,

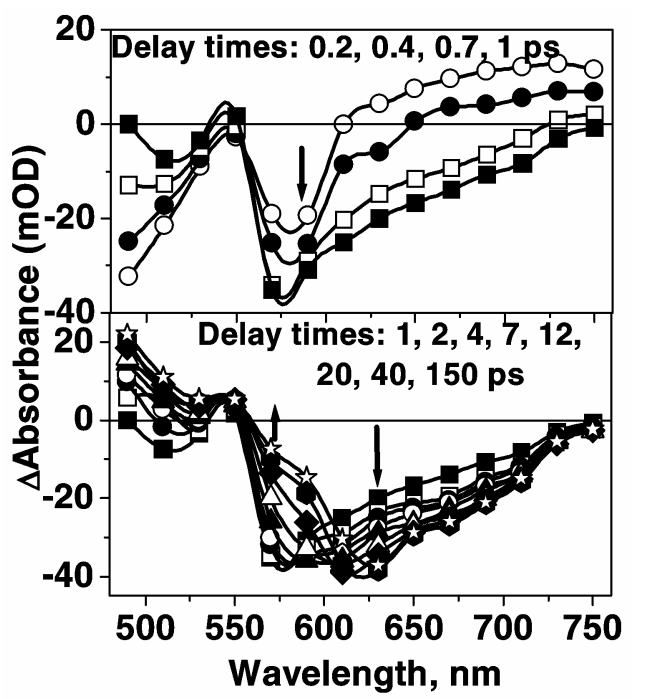

Figure 6. Time-resolved transient absorption spectra recorded following photoexcitation of MPAC in TFE using $400 \mathrm{~nm}$ laser pulses of $50 \mathrm{fs}$ duration. 
Table 1. Comparison of the relaxation time, $\tau_{2}$, which is assigned to the solvent reorganization process, with the viscosity $(\eta)$, longitudinal relaxation time $\left(\tau_{\mathrm{L}}\right)$, average solvation time $\left(\left\langle\tau_{\mathrm{s}}\right\rangle\right)$ and the rotational correlation time $\left(\tau_{\mathrm{OR}}\right)$ of the $\mathrm{OH}$ group of the corresponding solvent.

\begin{tabular}{lccccc}
\hline Solvents & $\eta(\mathrm{cP})$ & $\tau_{\mathrm{L}}(\mathrm{ps})$ & $\left\langle\tau_{\mathrm{s}}\right\rangle(\mathrm{ps})$ & $\tau_{\mathrm{OR}}(\mathrm{ps})$ & $\tau_{2}(\mathrm{ps})$ \\
\hline Acetonitrile & $0 \cdot 37$ & $0 \cdot 21$ & $0 \cdot 4$ & & 1.7 \\
DMSO & 1.99 & $2 \cdot 1$ & $1 \cdot 8$ & & $4 \cdot 5$ \\
Methanol & $0 \cdot 54$ & $9 \cdot 2$ & 5 & 5 & 12 \\
Ethanol & 1.1 & $27 \cdot 4$ & 16 & 18 & 22 \\
1-Propanol & 2 & 53 & 26 & 33 & 38 \\
1-Pentanol & 3.35 & 151 & 103 & & 58 \\
1-Octanol & $6 \cdot 1$ & 406 & 165 & & 65 \\
Cyclohexanol & 41.1 & 86 & 15 & & 66 \\
TFE & & & & & 27 \\
\hline
\end{tabular}

$\tau_{\mathrm{L}}$ values are taken from references 22 and $23 .\left\langle\tau_{\mathrm{s}}\right\rangle$ values are taken from references 24 and 25. $\tau_{\mathrm{OR}}$ values are taken from reference 26

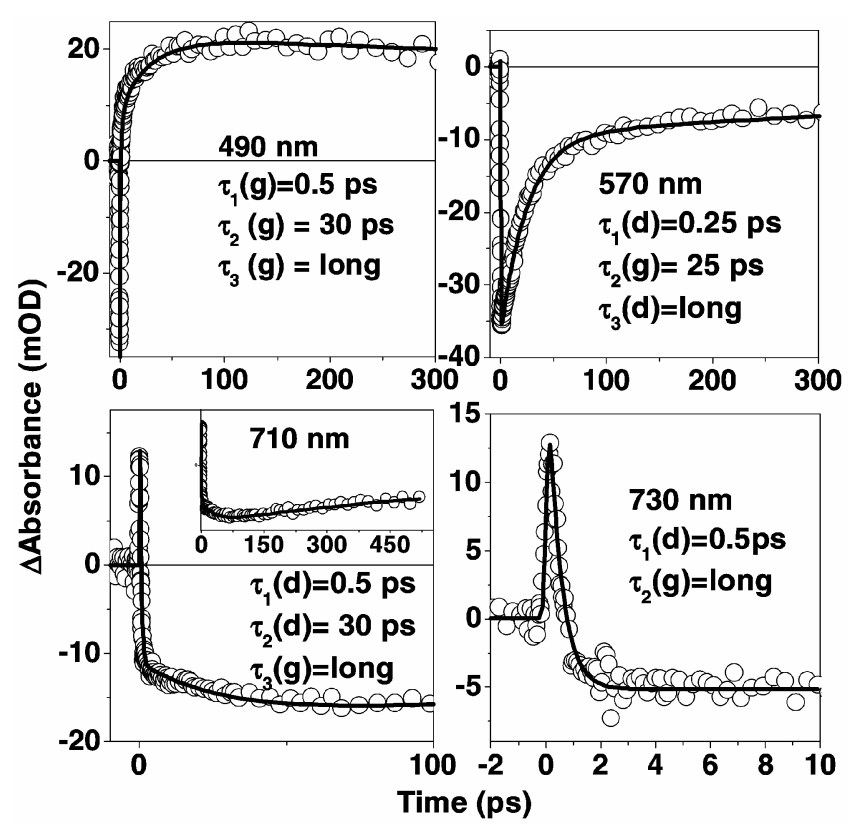

Figure 7. Temporal profiles of the transients monitored at a few selective wavelengths, following photoexcitation of MPAC in TFE using $400 \mathrm{~nm}$ light, along with the bestfit functions (solid lines). Lifetimes of different components are also shown in the figure.

namely the non-hydrogen-bonded form, which is formed following photoexcitation, to the hydrogenbonded complex of the $S_{1}$ state (vide infra).

Like in other protic solvents, each of the temporal profiles recorded in this solvent in the 490-710 nm region could be fitted with a function having three exponential terms. The ultrafast component (with the lifetime, $\tau_{1}=0.5 \mathrm{ps}$ ) is assigned to that of the $\mathrm{S}_{2}$ state and the second component with the lifetime, $\tau_{2}=27 \pm 3 \mathrm{ps}$, which is also independent of the monitoring wavelength, is assigned to that of the process of hydrogen-bond re-organization around the carbonyl oxygen atom leading to the formation of hydrogen-bonded form in the excited state. The lack of wavelength dependence of the lifetime of this component suggests the absence of the dipolar solvation process.

\section{Discussion}

Steady-state absorption and emission properties (\$3.1) as well as the spectral evolution and temporal dynamics of the excited states of MPAC as observed in the time-resolved studies (\$3.2) are remarkably different in aprotic and protic solvents. These facts suggest that the relaxation dynamics of the excited states are sensitive to the microscopic details of the solute-solvent interaction.

In polar aprotic solvents, namely acetonitrile and DMSO, the relaxation dynamics of the $\mathrm{S}_{1}$ state has been seen to be wavelength dependent and complex because of involvement of more than one transient species. In spite of the fact that the difference in dipole moment between the $S_{1}$ and $S_{0}$ states are small $(\sim 1.5 \mathrm{D})$, the ultrafast component with the lifetimes of 1.7 and $4.5 \mathrm{ps}$ in acetonitrile and DMSO, can be assigned to the ICT process, leading to a transient state having a fully conjugated polyene structure with no bond-length alternation. The reaction time increases with the average solvation time but remains slightly larger than the latter (table 1). These facts can be interpreted as indication that both solvent re-organization and internal re-structuring is 
involved in the ICT state formation. ${ }^{27}$ The intramolecular coordinate, which is involved in the charge transfer reaction, is possibly the torsional motion of the donor groups. On the other hand, the relaxation of the ICT state leads to photoisomerization and intersystem crossing to the triplet state. These processes have been shown earlier to be common in push-pull polyenes and the relative contribution of these two processes depend on the nature of the donor group, length of the polyene chain and the polarity of the solvent. ${ }^{27}$ Wavelength dependence of the temporal dynamics of the $S_{1}$ state of MPAC in polar aprotic solvents suggest the contribution of both these processes, which need more detailed investigation as a function of polarity and viscosity of the aprotic solvents.

A substantial amount of theoretical work has been devoted to understand the influence of solvent dynamics on the reaction rates. The most general conclusion is that solvent dynamics strongly affect the lifetimes, which is in contradiction to the transition state theory. ${ }^{28,29}$ In the case of solvent-controlled barrier crossing reactions, in which the reactant and the solvent are strongly coupled, dynamical solvent effects are usually discussed in terms of 'friction', $\zeta$, to account for the effect of the solvent on the lifetime $(\tau)$ of the reactant,,$^{30,31}$

$$
\tau=A \xi \exp (\Delta H / R T)
$$

The most important requirement for application of this equation to a particular reaction conducted in solution is to find out a measurable solvent parameter, which is the major factor affecting the rate of the reaction, while many other factors can also alter the rate of the same reaction. For reactions involving large amplitude motions, such as isomerization and dissociation reactions, collisional friction is important and the rates of these kinds of reactions can be correlated with the viscosity, $\eta$, of the medium.

$$
\ln \left(\tau_{2}\right)=\ln (\eta)+C+\left(\frac{\Delta H}{R T}\right)
$$

Figure 8a shows the plot of $\ln \left(\tau_{2}\right)$ vs $\ln (\eta)$ for the alcoholic solvents. We do not observe the linear correlation between these two parameters, as expected from (2). A qualitative examination of the data also reveals the lack of correlation of $\tau_{2}$ with solvent viscosities (table 1). For example, cyclohexanol has substantially higher viscosity $(41.1 \mathrm{cp})$ than that of 1-octanol (6.12 cp) but $\tau_{2}$ value in cyclohexanol and 1 -octanol is in the same range $(65 \pm 3 \mathrm{ps}$, table 1$)$. Therefore, $\tau_{2}$ is not due to any kind of dynamics related to conformation or configurational changes in the excited state.

For charge transfer reactions, dynamical solvent effect involves the dielectric friction, which arises in polar solvents due to electrostatic coupling between the solvent and the reactant. In this case, longitudinal relaxation time, $\tau_{\mathrm{L}}$, is taken to be proportional to the solvent friction. ${ }^{31}$

$$
\tau_{2}=A\left(\tau_{L}\right) \exp \left(\frac{\Delta H}{R T}\right)
$$

or

$$
\ln \left(\tau_{2}\right)=\ln \left(\tau_{L}\right)+A+\left(\frac{\Delta H}{R T}\right)
$$

If we assume that the enthalpy of the transition state, $\Delta H$, does not vary significantly due to change of solvents belonging to the same class, e.g. primary alcohols, we expect a linear correlation between $\ln \left(\tau_{2}\right)$ and $\ln \left(\tau_{\mathrm{L}}\right)(4)$. As shown in figure $8 \mathrm{~b}$, we find a perfect linear correlation between these two parameters. This confirms that the solvent re-organization around the excited solute molecule is solely responsible for the dynamics of the relaxation process of
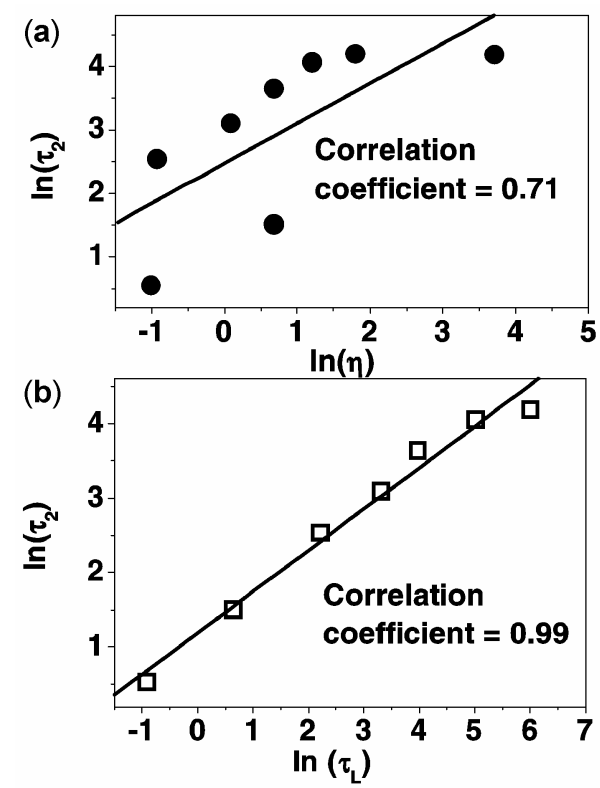

Figure 8. Plot of $\ln \left(\tau_{3}\right)$ vs $\ln (\eta)$ (a) and $\ln \left(\tau_{3}\right)$ vs $\ln \left(\tau_{\mathrm{L}}\right)$ (b). The solid lines represent the best-fit linear function. 
the $S_{1}$ state in the alcoholic solvents observed here. It is also reconfirmed that, in aprotic solvents too, the ultrafast component, $\tau_{2}$, which is associated with the relaxation process of the $\mathrm{S}_{1}$ state, is associated with the solvation of the ICT state.

In the case of protic solvents, the lifetime $\tau_{2}$ is a measure of the dynamics of specific interaction between the solute's electronic state and the solvent via intermolecular hydrogen bonding, which is accompanied by the re-organization of the hydrogenbonding network structure of the alcoholic solvent. Berg and co-workers revealed the interesting fact that the solvent dynamics affecting hydrogen bond reorganization time of the solvent is well-correlated with the dielectric relaxation time and the hydrogen bond lifetimes are similar to the longitudinal relaxation time, $\tau_{\mathrm{L}}{ }^{32}$ A broad range of hydrogen bond lifetimes have been reported in computer simulations. In general, the dielectric relaxation in alcohols is multi-exponential but the slowest component has generally been related to the time needed for the solvent hydrogen bond structure to breakdown and reform. ${ }^{33,34}$ Matsumoto and Gubbins simulated the average lifetime for the first breaking of the hydrogen bond in methanol is $0.9 \mathrm{ps}$, whereas the autocorrelation time of hydrogen bond, which measures the time for formation of a hydrogen bond and reorganization of the solvent hydrogen bond network structure to reach an equilibrium, is $11.8 \mathrm{ps} .^{35}$ This value is comparable to the value of $\tau_{\mathrm{L}}$ of methanol $(9 \cdot 2 \mathrm{ps})^{36}$

It is important to note that although we find linear correlations between $\tau_{2}$ and $\tau_{\mathrm{L}}$ in the alcoholic solvents (figure 8), these values do not match exactly with each other in the corresponding solvents (table 1). However, the larger difference between the $\tau_{\mathrm{R}}$ values determined here and the reported values of $\left\langle\tau_{\mathrm{s}}\right\rangle$ or $\tau_{\mathrm{L}}$ in the case of the alcoholic solvents with longer alkane chain may also possibly has arisen because of the contributions from both the thermalization processes, which mainly involve a change of the hydrogen bond length, as well as the translational component of the dipolar solvation. ${ }^{37-40}$

\section{Conclusion}

In this paper, we report the ultrafast relaxation dynamics of the $S_{2}$ and $S_{1}$ states of a ketocynaine dye, MPAC. Observation of $S_{2}$-fluorescence in ketocynanine dyes in rigid matrices at $77 \mathrm{~K}$ has been confirmed once again. Lifetime of the $S_{2}$ state in solution is about $0.5 \mathrm{ps}$ and nearly independent of solvent characteristics. However, the dynamics of the relaxation processes undergone by the $S_{1}$ state of MPAC is strongly dependent on the solvent characteristics. In aprotic solvents, the $\mathrm{S}_{1}$ state has been seen to undergo processes like intermolecular charge transfer, solvation as well as the photoisomerization. Yield of the intersystem crossing process is also quite significant in these kind of solvents, but nearly negligible in protic solvents. Solvent re-organization via intermolecular hydrogen bonding interaction is the major relaxation pathway for the $S_{1}$ state of MPAC in protic solvents. The $S_{1}$ state even forms hydrogen-bonded complex because of much stronger hydrogen-bonding interaction with TFE.

\section{Acknowledgements}

We thank Prof S Bagchi of the University of Burdwan for his gift of the dye. We are also grateful to Dr S K Sarkar, Head, Radiation and Photochmistry Division, BARC, for his constant encouragement.

\section{References}

1. Kessler M A and Wolfbeis O S 1991 Spectrochim. Acta. A47 187

2. (a) Banerjee D, Laha A K and Bagchi S 1995 Indian J. Chem. A34A 94; (b) Banerjee D, Laha A K and Bagchi S 1995 J. Photochem. Photobiol. A: Chem. 85 153; (c) Banerjee D, Mondal S, Ghosh, S and Bagchi S 1995 J. Photochem. Photobiol. A: Chem. 90 171; (d) Banerjee D, Das P K, Mondal S, Ghosh S and Bagchi S 1996 J. Photochem. Photobiol. A: Chem. 98 183; (e) Banerjee D and Bagchi S 1996 J. Photochem. Photobiol. A: Chem. 101 57; (f) Pramanik R, Das P K and Bagchi S 1999 J. Photochem. Photobiol. A: Chem. 124 135; (g) Pramanik R, Das P K and Bagchi S 2000 Phys. Chem. Chem. Phys. 2 4307; (h) Pramanik R, Das P K, Banerjee D and Bagchi S 2001 Chem. Phys. Lett. 341 507; (i) Shannigrahi M, Pramanik R and Bagchi S 2003 Spectrochim. Acta A59 2921; (j) Das P K Pramanik R, Banerjee D and Bagchi S 2000 Spectrochim. Acta A56 2763

3. Marcotte N and Fery-Forgues S $2000 \mathrm{~J}$. Photochem. Photobiol. A: Chem. 130133

4. Doroshenko A O and Pivovarenko V G $2003 \mathrm{~J}$. Photochem. Photobiol. A. Chem. 15655

5. Doroshenko A O, Bilokin M D and Pivovarenko V G 2004 J. Photochem. Photobiol. A: Chem. 163 95

6. Pivovarenko V G, Klueva A V, Doroshenko A O and Demchenko A P 2000 Chem. Phys. Lett. 325 389

7. (a) Lobnik A and Wolfbeis O S 1998 Analyst 123 2247; (b) Reichardt C 1994 Chem. Rev. 942319 
8. Doroshenko A O, Grigorovich A V, Posokhov E A, Pivovarenko A G and Demchenko A P $1999 \mathrm{~J}$. Mol. Eng. 8199

9. Doroshenko A O, Sychevskaya L B, Grygorovych, A V and Pivovarenko V G 2002 J. Fluoresc. 12451

10. Rurack K, Dekhtyar M L, Bricks J L, Resch-Genger U and Rettig W 1999 J. Phys. Chem. A103 9626

11. Barnabas M V, Liu A, Trifanac A D, Krougauz V V and Chang C T 199296212

12. Chambers W J and Eaton D F 1986 J. Imaging Sci. 13230

13. Baun M D and Henry C P 1972 Ger. Offen. 2 133; 315; Baun M D and Henry C P 1970 US Appl. 53686

14. Marcotte N, Ferry-Fogues S, Lavabre D, Marquet S and Pivovarenko V G 1999 J. Phys. Chem. 1033163

15. (a) Fabian J, Nakazami $H$ and Matsuoka M 1992 Chem. Rev. 92 1197; (b) Mishra A, Behera R K, Behera P K, Mishra B K and Behera G B 2000 Chem. Rev. 1001973

16. (a) Frank H A 2001 Arch. Biochem. Biophys. 385 53; (b) Poliska T and Sundstorm V 2004 Chem. Rev. 104 2021; (c) Koyama Y, Kuki M, Anderson P O and Gilbro T 1996 Photochem. Photobiol. 63243

17. (a) Lu D, Chen G, Perry J W and Beddard III W A 1994 J. Am. Chem. Soc. 116 10679; (b) Alain V, Thouin L, Bkanchard-Desce M, Gubler U, Bosshard C, Gunter P, Muller J, Fort A and Barzoukas M 1999 Adv. Mater. 11 1210; (c) Alain V, Blanchard-Dessce M, Ladous-Rak I and Zyss J 2000 Chem. Commun 353

18. Mondal J A, Ghosh $\mathrm{H} \mathrm{N}$, Mukherjee T, Palit D K 2005 J. Phys. Chem. A109 6836

19. Mondal J A, Ghosh H N, Ghanty T K, Mukherjee T and Palit D K 2006 J. Phys. Chem. A110 3432

20. El-Sayed M A 1963 J. Phys. Chem. 382834

21. Kovalenko S A, Ernsting N P and Ruthmann J 1997 J. Chem. Phys. 1063504

22. Simon J D 1988 Acc. Chem. Res. 21128

23. Davies M 1969 Dielectric properties and molecular behaviour (eds) N F Hill, W E Vaughan, A H Price and M Davies (London: Van Nostrand)
24. Lin Y and Jonah C D 1993 J. Phys. Chem. 97295

25. Horng H L, Dahl K, Jones II G and Maroncelli M 1999 Chem. Phys. Lett. 315363

26. Ludwig R and Zeidler M D 1966 Mol. Phys. 10451

27. Akemabn W, Laage D, Plaza P, Martin M M and Blanchard-Dessce M 2007 J. Phys. Chem. B106 (in press)

28. Hynes J T 1985 Theory of chemical reaction dynamics (ed.) M Bear (CRC: Boca Raton) vol IV

29. Hynes J T 1985 Annu. Rev. Phys. Chem. 36573

30. Berne B J, Borkovec M and Straub J E 2001 J. Phys. Chem. A105 6294

31. Kramers H 1940 Physica 7284

32. (a) Yu J and Berg M 1993 Chem. Phys. Lett. 208 315; (b) Benigno A J, Ahmed E and Berg M $1996 \mathrm{~J}$. Chem. Phys. 1047382

33. Garg S K and Smyth C P 1965 J. Phys. Chem. 69 1294

34. Bertolini D, Cassettari M and Salvetti G $1982 \mathrm{~J}$. Chem. Phys. 76325

35. Matsumoto M and Gubbins K $1990 \mathrm{~J}$. Chem. Phys. 931981

36. (a) Maroncelli M, MacInnis J and Fleming G. R. 1989 Science 243 1674; (b) Maroncelli M $1993 \mathrm{~J}$. Mol. Liq. 57 1; (c) Maroncelli M, Castner Jr E W, Bagchi B and Fleming G R 1988 Faraday Discuss. Chem. Soc. 85 199; (d) Horng M L, Gardecki J A, Papazyan A and Maroncelli M 1995 J. Phys. Chem. 9917311

37. Kenney-Wallace G A, Paone S and Kalpouzos C 1988 Faraday Discuss. Chem. Soc. 85185

38. Biswas R, Nandi N and Bagchi B 1997 J. Phys. Chem. B101 2968

39. (a) Gale G M, Gallot G, Hache F, Lascoux N, Bratos S and Leicknam J-C 1999 Phys. Rev. Lett. 82 1086; (b) Woutersen S and Bakker H J 1999 Phys. Rev. lett. 832707

40. Lock A J, Woutersen S and Bakker H J $2001 \mathrm{~J}$. Phys. Chem. A105 1238

41. Joo T, Jia $Y, Y u J-Y$, Lang $M J$ and Fleming $G R$ 1996 J. Chem. Phys. 1046089 